\title{
Accurate Approximations for the Complex Error Function with Small Imaginary Argument
}

\author{
S. M. Abrarov ${ }^{1} \&$ B. M. Quine ${ }^{1,2}$ \\ ${ }^{1}$ York University, Dept. Earth and Space Science and Engineering, 4700 Keele Street, Toronto, M3J 1P3, Canada. \\ 2 York University, Dept. Physics and Astronomy, 4700 Keele Street, Toronto, M3J 1P3, Canada. \\ Correspondence: S. M. Abrarov, ESSE, York University, 4700 Keele Street, Toronto, M3J 1P3, Canada. E-mail: \\ absanj@gmail.com
}

Received: November 3, 2014 Accepted: November 20, 2014 Online Published: January 19, 2015

doi:10.5539/jmr.v7n1p44 URL: http://dx.doi.org/10.5539/jmr.v7n1p44

\begin{abstract}
In this paper we present two efficient approximations for the complex error function $w(z)$ with small imaginary argument $\operatorname{Im}[z]<<1$ over the range $0 \leq \operatorname{Re}[z] \leq 15$ that is commonly considered difficult for highly accurate and rapid computation. These approximations are expressed in terms of the Dawson's integral $F(x)$ of real argument $x$ that enables their efficient implementation in a rapid algorithm. The error analysis we performed using the random input numbers $x$ and $y$ reveals that in the real and imaginary parts the average accuracy of the first approximation exceeds $10^{-9}$ and $10^{-14}$, while the average accuracy of the second approximation exceeds $10^{-13}$ and $10^{-14}$, respectively. The first approximation is slightly faster in computation. However, the second approximation provides excellent high-accuracy coverage over the required domain.
\end{abstract}

Keywords: complex error function, Voigt function, Faddeeva function, Kramp function, Dawson's integral

\section{Introduction}

The complex error function, also known as the Faddeeva function or the Kramp function, can be defined as (Faddeeva \& Terent'ev, 1961; Abramowitz \& Stegun, 1972; Armstrong \& Nicholls 1972; Poppe \& Wijers 1990a, 1990b; Schreier, 1992)

$$
\begin{aligned}
w(z) & =e^{-z^{2}}[1-\operatorname{erf}(-i z)] \\
& =e^{-z^{2}}\left(1+\frac{2 i}{\sqrt{\pi}} \int_{0}^{z} e^{t^{2}} d t\right),
\end{aligned}
$$

where $z=x+i y$ is the complex argument. Using the Fourier transforms, the real and imaginary parts of the complex error function (1) can be represented as (Armstrong \& Nicholls 1972; Srivastava \& Chen 1992; Pagnini \& Mainardi, 2010)

$$
K(x, y)=\frac{1}{\sqrt{\pi}} \int_{0}^{\infty} \exp \left(-t^{2} / 4\right) \exp (-y t) \cos (x t) d t, \quad y>0
$$

and

$$
L(x, y)=\frac{1}{\sqrt{\pi}} \int_{0}^{\infty} \exp \left(-t^{2} / 4\right) \exp (-y t) \sin (x t) d t, \quad y>0,
$$

respectively. The real part of the complex error function $K(x, y)$ is known as the Voigt function (Letchworth \& Benner, 2007), widely used in many fields of Physics, Astronomy and Chemistry (Christensen et. al, 2012; Sonnenschein et. al, 2012; Berk, 2013; Quine \& Abrarov, 2013). Combining the real (2) and imaginary (3) parts together as $w(x, y)=K(x, y)+i L(x, y)$ yields

$$
w(x, y)=\frac{1}{\sqrt{\pi}} \int_{0}^{\infty} \exp \left(-t^{2} / 4\right) \exp (-y t) \exp (i x t) d t, \quad y>0 .
$$


None of the integrals above can be taken analytically in closed form. Consequently, the complex error function must be computed numerically.

It should be noted that from identity (McKenna, 1984; Zaghloul \& Ali, 2011)

$$
w(-z)=2 e^{-z^{2}}-w(z),
$$

it follows that only positive arguments $x$ and $y$ are sufficient in order to cover the entire complex plane. Therefore, we will imply further that both input parameters $x$ and $y$ are always positive.

When the argument $z$ is large enough by absolute value, say $|x+i y|>15$, the truncation of the Laplace continued fraction (see Gautschi, 1970; Jones \& Thron, 1988, see also Poppe \& Wijers 1990a, 1990b)

$$
w(z)=\frac{\mu_{0}}{z-} \frac{1 / 2}{z-} \frac{1}{z-} \frac{3 / 2}{z-} \frac{2}{z-} \frac{5 / 2}{z-} \cdots, \quad \mu_{0} \equiv i / \sqrt{\pi},
$$

can be effectively used for high-accuracy and rapid computation of the complex error function $w(z)$.

There are several approximations such as the Chiarella and Reichel approximation (equation (15) in Abrarov \& Quine, 2014), the Weideman's rational approximation (equation (38-I) in Weideman, 1994), the exponential series approximation (equation (14) in Abrarov \& Quine, 2011 and its modification (3) in Abrarov \& Quine, 2012) and the rational approximation (equation (14) in Abrarov \& Quine, 2014) that provide highly accurate and rapid calculation for the complex error function within domain $0 \leq x \leq 15$ and $10^{-6} \leq y \leq 15$.

However, the highly accurate and simultaneously rapid computation of $w(z)$ at $y<<1$ and $|x+i y| \leq 15$ still remains problematic (Armstrong, 1967; Amamou et. al, 2013). Different approaches have been implemented to overcome this problem. For example, Zaghloul and Ali (2011) developed Algorithm 916 that can cover accurately this domain. In the rapid algorithm developed by Karbach et al. (2014) this domain is covered by using the exponential series approximation (equation (14) in Abrarov \& Quine, 2011), the Taylor expansion series near singularities at $z_{n}=n \pi / \tau_{m}$ ( $n$ is the index integer and $\tau_{m}$ is the integration cutoff) and the Laplace continued fraction. In our recent work we proposed to cover this domain by using the master-slave algorithm (Abrarov \& Quine, 2014) (see also Matlab source code in Matlab Central, file ID: \#47801 (2014), where the master-slave approach has been implemented to cover it). In this paper we report two new approximations that also effectively resolve this problem for accurate and rapid computation.

\section{Approximations for small $y$}

A simplest way to approximate the complex error function $w(x, y<<1)$ is to use the Maclaurin expansion series near the point $y=0$ (see for example two approximations (A.4) and (A.5) in Appendix A). However, this method results in a moderate accuracy that does not exceed $10^{-6}$ in the range of a concern $|x+i y| \leq 15$ at $y<<1$.

In order to obtain more efficient approximations, we may attempt to find an appropriate representation of the complex error function $w(z)$. Let us rewrite the equation of the complex error function (4) as

$$
w(x, y)=\frac{e^{y^{2}}}{\sqrt{\pi}} \int_{0}^{\infty} e^{-(t+2 y)^{2} / 4} e^{i x t} d t .
$$

Change of the variable $u=t+2 y$ in equation above excludes the parameter $y$ from the integrand:

$$
\begin{aligned}
w(x, y) & =\frac{e^{y^{2}}}{\sqrt{\pi}} \int_{2 y}^{\infty} e^{-u^{2} / 4} e^{i x(u-2 y)} d u \\
& =\frac{e^{y^{2}-2 i x y}}{\sqrt{\pi}} \int_{2 y}^{\infty} e^{-u^{2} / 4} e^{i x u} d u .
\end{aligned}
$$

Consequently, this equation can be rearranged as

$$
w(x, y)=\frac{e^{y^{2}-2 i x y}}{\sqrt{\pi}}\left(\int_{0}^{\infty} e^{-u^{2} / 4} e^{i x u} d u-\int_{0}^{2 y} e^{-u^{2} / 4} e^{i x u} d u\right) .
$$


The first integral in the equation above is

$$
\int_{0}^{\infty} e^{-u^{2} / 4} e^{i x u} d u=e^{-x^{2}} \sqrt{\pi}+2 i F(x)
$$

where

$$
F(x)=\frac{1}{2} \int_{0}^{\infty} e^{-u^{2} / 4} \sin (x u) d u
$$

is the Dawson's integral. As the argument $x$ in $F(x)$ is real, its computation is not difficult and several efficient approximations that can provide rapid and highly accurate computation are reported in literature (Rybicki, 1989; McCabe, 1974; Cody et. al, 1970). Furthermore, the latest versions of Matlab support the built-in function dawson $(x)$ that computes very rapidly the Dawson's integral of real argument.

The advantage of the equation (5) is that the integration range in the second integral at $y<<1$ is very narrow. Consequently, the second integral in equation (5) makes only a minor contribution to the complex error function $w(z)$. As a result, this minimizes the error in computation.

The representation of the complex error function in form of (5) provides enormous flexibility to approximate it. Here we show two efficient derivations that directly follow from equation (5).

As the upper limit of the second integral of equation (5) is finite, its evaluation is not straightforward (see Appendix B). However, this problem can be overcome by Maclaurin expansion of the exponential function:

$$
e^{-u^{2} / 4}=1-\frac{u^{2}}{4}+\frac{u^{4}}{32}-\frac{u^{6}}{384}+O\left(u^{8}\right) .
$$

The first approximation is obtained by substituting only the first term of this expansion into the second integral of equation (5) that yields

$$
w(x, y<<1) \approx e^{(i x-y)^{2}}\left[1+\frac{i e^{x^{2}}}{\sqrt{\pi}}\left(2 F(x)-\frac{1-e^{2 i x y}}{x}\right)\right] .
$$

We will refer to this equation as the basic approximation. This equation is highly accurate while $x \lesssim 3$.

The second approximation is obtained by substituting the first two terms of the expansion above into second integral of the equation (5):

$$
\begin{aligned}
w(x, y<<1) & \approx e^{(i x-y)^{2}}\left[1+\frac{i e^{x^{2}}}{\sqrt{\pi}}\right. \\
& \left.\times\left(2 F(x)-\frac{x^{2}+0.5+e^{2 i x y}\left(x^{2}\left(y^{2}-1\right)+i x y-0.5\right)}{x^{3}}\right)\right] .
\end{aligned}
$$

Further, we will refer to this equation as the main approximation.

Although the basic approximation (6) is slightly faster in computation, the main approximation (7) provides essentially higher accuracy as it will be shown in the section 4 .

\section{Algorithmic implementation}

Approximations (6) and (7) cannot be employed directly in the computation flow since at $x \rightarrow 0$ the denominators $x$ in approximation (6) and $x^{3}$ in approximation (7) blow up the ratios resulting to computational overflow. However, this problem is very easy to resolve by using, for example, the following supplement:

$$
w(x<<1, y<<1) \approx\left(1-(x+i y)^{2}\right)\left(1+\frac{2 i(x+i y)}{\sqrt{\pi}}\right) .
$$

In particular, the computation flow for the basic and the main approximations can be maintained as

$$
w(x, y<<1) \approx\left\{\begin{aligned}
\text { Eq.(6) or Eq.(7), } & 10^{-4}<x \leq 15 \cap y \leq 10^{-6} \\
\text { Eq.(8), } & 0 \leq x \leq 10^{-4} \cap y \leq 10^{-6} .
\end{aligned}\right.
$$


Thus, according to this scheme if $x>10^{-4}$ the complex error function is computed either by equation (6) or by equation (7). Otherwise, if $x \leq 10^{-4}$ it is computed by equation (8).

The approximation (8) had been used already in our recently published Matlab source code (Matlab Central, file ID: \#47801, 2014) to resolve a similar problem that occurs at $|x+i y| \rightarrow 0$. The derivation of the approximation (8) is shown in Appendix A.

\section{Error analysis}

Define the relative errors for the real and imaginary parts of the complex error function in forms

$$
\Delta_{\operatorname{Re}}=\left|\frac{\operatorname{Re}[w(z)]-\operatorname{Re}\left[w_{\text {ref. }}(z)\right]}{\operatorname{Re}\left[w_{\text {ref. }}(z)\right]}\right|
$$

and

$$
\Delta_{\operatorname{Im}}=\left|\frac{\operatorname{Im}[w(z)]-\operatorname{Im}\left[w_{\text {ref. }}(z)\right]}{\operatorname{Im}\left[w_{\text {ref. }}(z)\right]}\right|,
$$

respectively, where $w_{\text {ref. }}(z)$ is the reference. The highly accurate reference values can be obtained according to equation (1) by using the latest versions of Wolfram Mathematica that supports error function of complex argument.

Figure 1a shows the logarithm of relative error for the real part of the basic approximation (6) in the domain $10^{-4} \leq$ $x \leq 15$ and $y \leq 10^{-6}$. As we can see, at $x \lesssim 3$ the approximation (6) is highly accurate and provides accuracy better than $10^{-12}$. Although the accuracy of the approximation (6) deteriorates as $x$ increases, it, nevertheless, still remains high and better than $10^{-9}$.

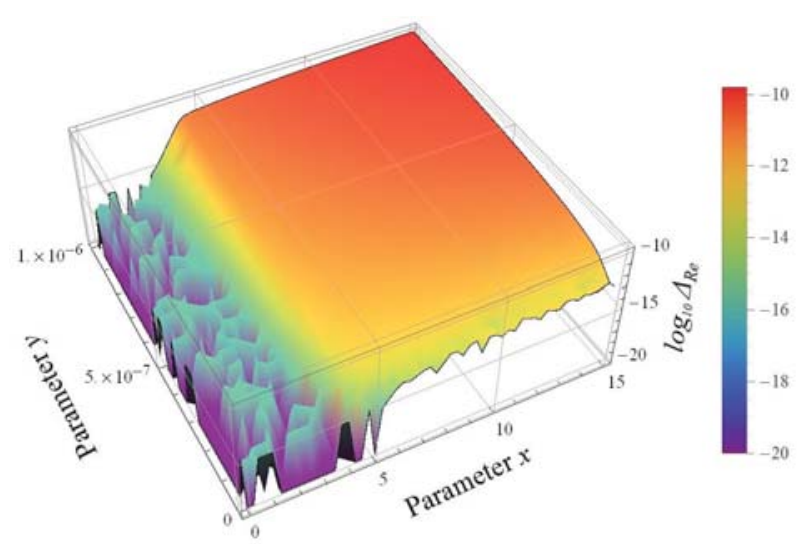

Figure 1a. Logarithm of relative error $\log _{10} \Delta_{\mathrm{Re}}$ for the real part of the basic approximation (6) over the domain $10^{-4} \leq x \leq 15$ and $y \leq 10^{-6}$.

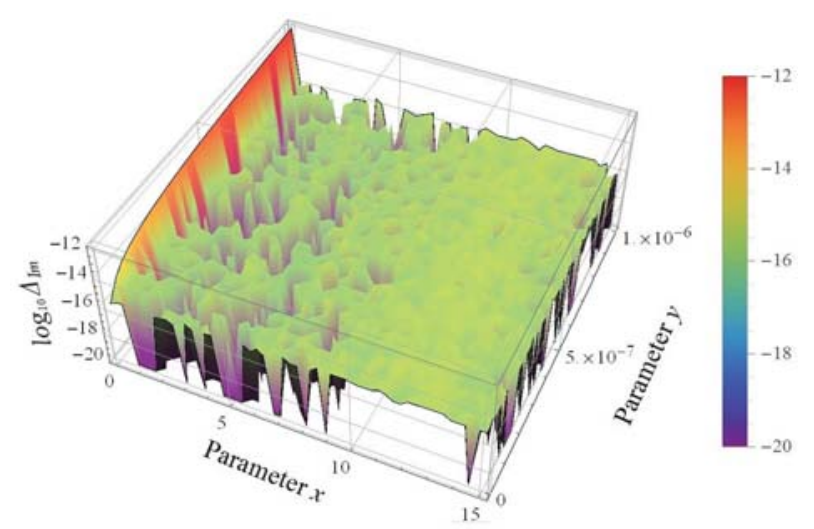

Figure 1b. Logarithm of relative error $\log _{10} \Delta_{\operatorname{Im}}$ for the imaginary part of the basic approximation (6) over the domain $10^{-4} \leq x \leq 15$ and $y \leq 10^{-6}$. 
Figure $1 \mathrm{~b}$ illustrates the logarithm of relative error for the imaginary part of the basic approximation (6) in the domain $10^{-4} \leq x \leq 15$ and $y \leq 10^{-6}$. As one can see, the imaginary part of the approximation (6) is highly accurate. Specifically, the accuracy is better than $10^{-14}$ almost over all of the domain. However, at small $x \lesssim 10^{-3}$ the accuracy deteriorates as it can be seen by red color along the left edge of $y$-axis.

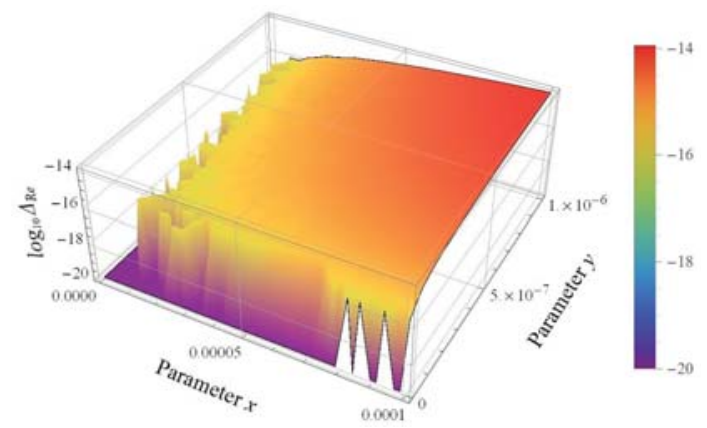

Figure 2a. Logarithm of relative error $\log _{10} \Delta_{\mathrm{Re}}$ for the real part of the supplementary approximation (8) over the small domain $0 \leq x \leq 10^{-4}$ and $y \leq 10^{-6}$.

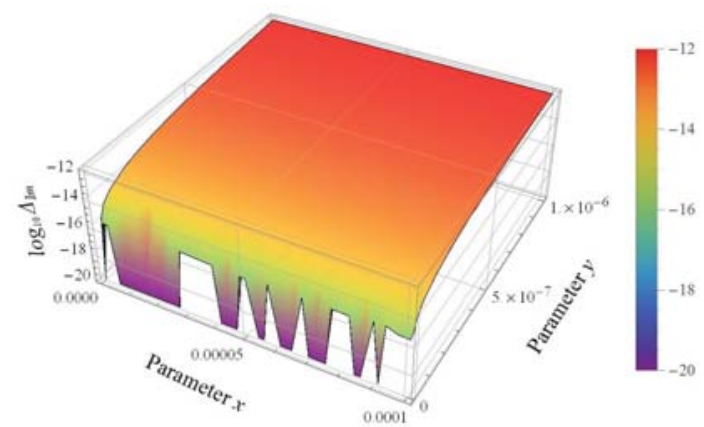

Figure 2b. Logarithm of relative error $\log _{10} \Delta_{\mathrm{Im}}$ for the imaginary part of the supplementary approximation (8) over the small domain $0 \leq x \leq 10^{-4}$ and $y \leq 10^{-6}$.

Figure 2a illustrates the logarithm of relative error $\log _{10} \Delta_{\mathrm{Re}}$ for the real part of the supplementary approximation (8) over the small domain $0 \leq x \leq 10^{-4}$ and $y \leq 10^{-6}$. One can see that the real part over this domain is highly accurate and better than $10^{-13}$.

Figure $2 \mathrm{~b}$ depicts the logarithm of relative error $\log _{10} \Delta_{\mathrm{Im}}$ for the imaginary part of the supplementary approximation (8) over the small domain $0 \leq x \leq 10^{-4}$ and $y \leq 10^{-6}$. The accuracy of the imaginary part is also high and better than $10^{-12}$.

From Figs. 1a and $1 \mathrm{~b}$ we can conclude that the basic approximation (6) alone may be used practically as the accuracy better than $10^{-9}$ is more than enough for the most applications.

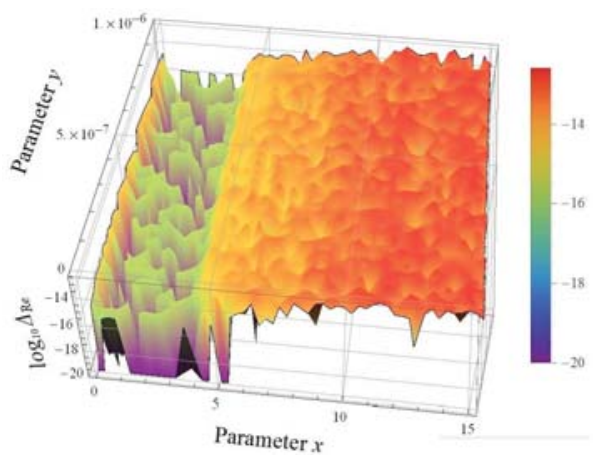

Figure 3a. Logarithm of relative error $\log _{10} \Delta_{\mathrm{Re}}$ for the real part of the main approximation (7) over the domain $10^{-4} \leq x \leq 15$ and $y \leq 10^{-6}$. 


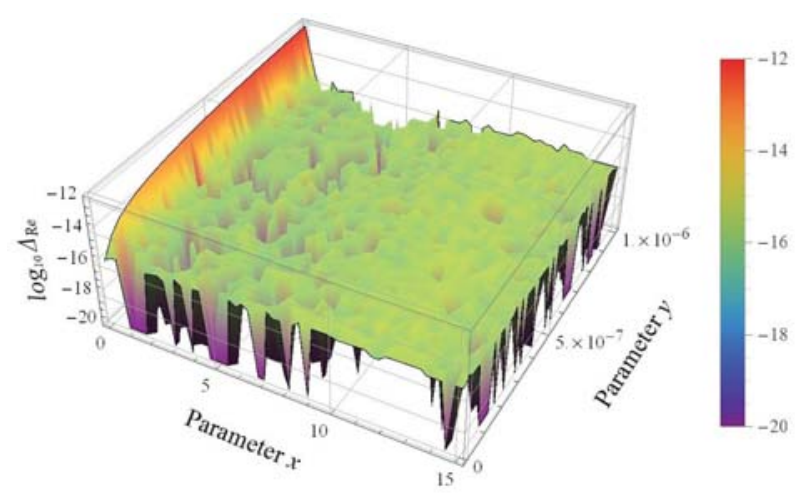

Figure 3b. Logarithm of relative error $\log _{10} \Delta_{\operatorname{Im}}$ for the imaginary part of the main approximation (7) over the domain $10^{-4} \leq x \leq 15$ and $y \leq 10^{-6}$.

The accuracy of the complex error function $w(x, y<<1)$ can be further improved by using the main approximation (7). Figure 3a depicts the logarithm of relative error for the real part of the approximation (7) in the domain $10^{-4} \leq x \leq 15$ and $y \leq 10^{-6}$. As it can be seen from this figure, in the real part the accuracy of the approximation (7) is better than $10^{-13}$.

Figure $3 \mathrm{~b}$ shows the logarithm of relative error for the imaginary part of the main approximation (7) in the domain $10^{-4} \leq x \leq 15$ and $y \leq 10^{-6}$. Comparing Figs. $3 \mathrm{~b}$ with $1 \mathrm{~b}$ one can observe that the graphs are nearly same. This signifies that the behavior of the basic and the main approximations resemble in their imaginary parts. Similar to Fig. 1b, the accuracy in the imaginary part is better than $10^{-14}$ almost over all domain.

To determine the average accuracy for both approximations, we performed calculations with randomly chosen input variables $x$ and $y$. It has been found that in the real and imaginary parts the basic approximation (6) provides average accuracy exceeding $10^{-9}$ and $10^{-14}$, while the main approximation (7) provides average accuracy exceeding $10^{-13}$ and $10^{-14}$, respectively.

\section{Application of approximations}

Consider an application of the approximations (6) or (7). As we have shown in our recent publication (Abrarov \& Quine, 2014), the approximation of the complex error function

$$
w(z) \approx \frac{e^{\sigma^{2}}}{\tau_{m}(\sigma-i z)}+\sum_{n=1}^{N} \frac{A_{n}(\sigma-i z)+B_{n}}{n^{2} \pi^{2}+\tau_{m}^{2}(\sigma-i z)^{2}}, \quad \operatorname{Im}[z]>0,
$$

where $N=23, \tau_{m}=12, \sigma=2, A_{n}=2 \tau_{m} e^{\sigma^{2}-n^{2} \pi^{2} / \tau_{m}^{2}} \cos \left(2 n \pi \sigma / \tau_{m}\right)$ and $B_{n}=2 n \pi e^{\sigma^{2}-n^{2} \pi^{2} / \tau_{m}^{2}} \sin \left(2 n \pi \sigma / \tau_{m}\right)$, covers almost all first quadrant with high-accuracy except the narrow domain $x>4 \cap y \lesssim 10^{-6}$ along $x$-axis. Although the multiple precision arithmetic can be applied to cover the remaining domain $x>4 \cap y \lesssim 10^{-6}$, it requires a special library of the function files that has to be properly installed and validated making its application very inconvenient. Recently, in order to resolve such a problem we proposed the master-slave approach (Abrarov \& Quine, 2014).

For future prospects, we can also use the approximations (6) or (7) as an efficient alternative to resolve this problem. In particular, when the high-accuracy, say better than $10^{-13}$, is required, the computation flow can be maintained as

$$
w(x, y) \approx \begin{cases}\text { Eq.(9), } & \{x, y\} \notin x>4 \cap y \leq 10^{-6} \\ \text { Eq.(7), } & \{x, y\} \in x>4 \cap y \leq 10^{-6} .\end{cases}
$$

As we can see, this scheme does not utilize the approximation (8), as the equation (9) itself provides high-accuracy when the both parameters $x<<1$ and $y<<1$ are small. As the accuracy better than $10^{-9}$ is sufficient for the most practical applications, the main approximation (7) can be replaced by basic approximation (6) to cover the narrow domain $x>4 \cap y \lesssim 10^{-6}$. The more detailed description, analysis and development of this application approach will be shown in our future work.

\section{Conclusion}

We present two efficient approximations for the complex error function that can be used for computation at small $y<<1$. As these approximations are expressed in terms of the Dawson's integral $F(x)$ of real argument $x$, they 
are rapid in computation. Error analysis performed with randomly taken input variables $x$ and $y$ reveals that in the real and imaginary parts the basic approximation (6) provides average accuracy exceeding $10^{-9}$ and $10^{-14}$, while the main approximation (7) provides average accuracy exceeding $10^{-13}$ and $10^{-14}$, respectively. Although the basic equation (6) is slightly faster in computation, the main approximation (7) provides excellent high-accuracy coverage.

\section{Acknowledgments}

This work is supported by National Research Council of Canada, Thoth Technology Inc. and York University. The authors wish to thank Prof. Ian McDade and Dr. Brian Solheim for discussions and constructive suggestions.

\section{Appendix A}

Consider the following Maclaurin expansions at $y=0$ :

$$
\begin{gathered}
e^{-(x+i y)^{2}}=e^{-x^{2}}-2 i x y e^{-x^{2}}+O\left(y^{2}\right), \\
\operatorname{erf}(-i(x+i y))=-i \operatorname{erfi}(x)+\frac{2 y}{\sqrt{\pi}} e^{x^{2}}+O\left(y^{2}\right)
\end{gathered}
$$

and

$$
\begin{aligned}
w(x, y) & \equiv e^{-(x+i y)^{2}}[1-\operatorname{erf}(-i(x+i y))] \\
& =e^{-x^{2}}(1+i \operatorname{erfi}(x))+2 y\left(x e^{-x^{2}} \operatorname{erfi}(x)-i x e^{-x^{2}}-\frac{1}{\sqrt{\pi}}\right)+O\left(y^{2}\right),
\end{aligned}
$$

Substituting (A.1) and (A.2) into identity (1) we obtain

$$
w(x, y<<1) \approx(1-2 i x y)\left(e^{-x^{2}}(1+\operatorname{erf}(i x))-\frac{2 y}{\sqrt{\pi}}\right),
$$

while equation (A.3) immediately results in

$$
w(x, y<<1) \approx e^{-x^{2}}(1+\operatorname{erf}(i x))(1-2 i x y)-\frac{2 y}{\sqrt{\pi}} .
$$

The approximations (A.4) and (A.5) are rapid in computation because the error function of imaginary argument erf $(i x)$ can be expressed in terms of the Dawson's integral of real argument $F(x)$ as follows

$$
\operatorname{erf}(i x) \equiv \frac{2 i}{\sqrt{\pi}} e^{x^{2}} F(x) .
$$

However, the approximations (A.4) and (A.5) are moderately accurate and should be used when the accuracy requirement does not exceed $10^{-6}$.

Same technique can be used to obtain approximation for $w(x<<1, y<<1)$ from the expansions near the point $z=0$ :

$$
\exp \left(-z^{2}\right)=1-z^{2}+O\left(z^{4}\right)
$$

and

$$
\operatorname{erf}(-i z)=-\frac{2 i z}{\sqrt{\pi}}+O\left(z^{3}\right)
$$

In particular, substituting these expansions into identity (1) leads to the approximation (8).

\section{Appendix B}

The second integral of equation (5) can be expressed as

$$
\int_{0}^{2 y} e^{-u^{2} / 4} e^{i x u} d u=\sqrt{\pi} e^{-x^{2}}[\operatorname{erf}(i x)-\operatorname{erf}(i x-y)]
$$

The argument in the first error function erf $(i x)$ is purely imaginary and, therefore, it can be expressed in term of the Dawson's integral of real argument. However, since the argument in the second error function erf $(i x-y)$ is 
complex, it needs a numerical solution (see for example equations (10) and (11) in Zaghloul \& Ali (2011)). When the high accuracy is not required, the rapid approximation for the error function can obtained, for example, by rearranging the expansion series (A.2) as

$$
\begin{aligned}
\operatorname{erf}(i x-y) & \approx \operatorname{ierfi}(x)-\frac{2 y}{\sqrt{\pi}} e^{x^{2}} \\
& \equiv \operatorname{erf}(i x)-\frac{2 y}{\sqrt{\pi}} e^{x^{2}}, \quad y<<1 .
\end{aligned}
$$

We can also approximate this integral by taking into account that $y<<1$. In particular, for small interval we can write $d u \approx 2 y$ and take the mid-point in that interval equal to $y$ :

$$
\int_{0}^{2 y} e^{-u^{2} / 4} e^{i x u} d u \approx 2 y e^{-y^{2} / 4} e^{i x y}
$$

Alternatively, according to the trapezoidal rule we get

$$
\int_{0}^{2 y} e^{-u^{2} / 4} e^{i x u} d u \approx y\left(1+e^{-y^{2}} e^{2 i x y}\right) .
$$

Once again, these approximations should be applied only when the high-accuracy is not a concern in computation. The second integral from equation (5) can be approximated more precisely by taking integration by parts

$$
\int_{0}^{2 y} e^{-u^{2} / 4} e^{i x u} d u=\left.\sqrt{\pi} \operatorname{erf}\left(\frac{u}{2}\right) e^{i x u}\right|_{0} ^{2 y}-\sqrt{\pi} i x \int_{0}^{2 y} \operatorname{erf}\left(\frac{u}{2}\right) e^{i x u} d u
$$

and then substituting the first few terms from the following expansion

$$
\operatorname{erf}\left(\frac{u}{2}\right)=\frac{u}{\sqrt{\pi}}-\frac{u^{3}}{12 \sqrt{\pi}}+\frac{u^{5}}{160 \sqrt{\pi}}-\frac{u^{7}}{2688 \sqrt{\pi}}+O\left(u^{9}\right)
$$

into the right integral of equation (B.1). We will consider the simplest case when only the first term from this expansion is substituted. Following this procedure and using equation (5) we have

$$
\begin{aligned}
w(x, y<<1) & \approx e^{(i x-y)^{2}}\left[1+\frac{i e^{x^{2}}}{\sqrt{\pi}}\right. \\
& \left.\times\left(2 F(x)-\frac{1-e^{2 i x y}(1+i x(\sqrt{\pi} \operatorname{erf}(y)-2 y))}{x}\right)\right] .
\end{aligned}
$$

The consistency between approximations (6) and (B.2) becomes evident from the fact that erf $(y<<1) \approx 2 y / \sqrt{\pi}$. Although approximation (B.2) is more accurate than the basic approximation (6), it is slightly slower in computation at large size of the input array due to presence of the error function erf (y). Since the approximation (B.2) provides nearly same accuracy as the main approximation (7), both of them can be used when the high-accuracy computation is required.

\section{References}

Abramowitz, M., \& Stegun, I. A. (1972). Error function and Fresnel integrals. Handbook of mathematical functions with formulas, graphs, and mathematical tables. $9^{\text {th }}$ Ed. Dover, New York, 297-309.

Abrarov, S. M., \& Quine, B. M. (2011). Efficient algorithmic implementation of the Voigt/complex error function based on exponential series approximation. Appl. Math. Comput., 218(5), 1894-1902. http://doi.org/10.1016/j.amc.2011.06.072

Abrarov, S. M., \& Quine, B. M. (2012). On the Fourier expansion method for highly accurate computation of the Voigt/complex error function in a rapid algorithm. ArXiv:1205.1768. http://arxiv.org/pdf/1205.1768v1.pdf 
Abrarov, S. M., \& Quine, B. M. (2014). Master-slave algorithm for highly accurate and rapid computation of the Voigt/complex error function. J. Math. Research, 6(2), 104-119. http://doi.org/10.5539/jmr.v6n2p104

Amamou, H., Ferhat, B., \& Bois, A. (2013). Calculation of the Voigt function in the region of very small values of the parameter a where the calculation is notoriously difficult, Amer. J. Anal. Chem., 4, 725-731. http://doi.org/10.4236/ajac.2013.412087

Armstrong, B. H. (1967). Spectrum line profiles: the Voigt function, J. Quant. Spectrosc. Radiat. Transfer. 7(1), 61-88. http://doi.org/10.1016/0022-4073(67)90057-X

Armstrong, B. H., \& Nicholls, B. W. (1972). Emission, absorption and transfer of radiation in heated atmospheres. Pergamon Press, New York, 1972.

Berk, A. (2013). Voigt equivalent widths and spectral-bin single-line transmittances: Exact expansions and the MODTRAN@5 implementation. J. Quantit. Spectrosc. Radiat. Transfer, 118, 102-120. http://dx.doi.org/10.1016/j.jqsrt.2012.11.026

Christensen, L. E., Spiers, G. D., Menzies, R. T., \& Jacob, J. C. (2012). Tunable laser spectroscopy of $\mathrm{CO}_{2}$ near $2.05 \mu \mathrm{m}$ : Atmospheric retrieval biases due to neglecting line-mixing. J. Quantit. Spectrosc. Radiat. Transfer, 113(10), 739-748. http://doi.org/10.1016/j.jqsrt.2012.02.031

Cody, W. J., Paciorek, K. A., \& Thacher, H. C. (1970). Chebyshev approximations for Dawson's integral. Math. Comp. 24, 171-178. http://dx.doi.org/10.1090/S0025-5718-1970-0258236-8

Faddeyeva, V. N., \& Terent'ev, N. M. (1961). Tables of the probability integral for complex argument. Pergamon Press, Oxford, 1961.

Gautschi, W. (1970). Efficient computation of the complex error function. SIAM J. Numer. Anal., 7(1), $187-198$. http://dx.doi.org/10.1137/0707012

Jones, W. B., \& Thron, W. J. (1988). Continued fractions in numerical analysis. Appl. Num. Math., 4(2-4), 143-230. http://doi.org/10.1016/0168-9274(83)90002-8

Karbach, T. M., Raven, G., \& Schiller, M. (2014). Decay time integrals in neutral meson mixing and their efficient evaluation. ArXiv:1407.0748. http://arxiv.org/pdf/1407.0748v1.pdf

Letchworth, K. L., \& Benner, D. C. (2007). Rapid and accurate calculation of the Voigt function. J. Quantit. Spectrosc. Radiat. Transfer, 107(1), 173-192. http://dx.doi.org/10.1016/j.jqsrt.2007.01.052

Matlab Central, file ID: \#47801, submitted on Sept. 10, 2014. The Matlab source code for computation of the Voigt/complex error function can be downloaded here: http://www.mathworks.com/matlabcentral/fileexchange/47801-the-voigt-complex-error-function

McCabe, J. H. (1974). A continued fraction expansion with a truncation error estimate for Dawson's integral, Math. Comp. 28(127), 811-816. http://dx.doi.org/10.1090/S0025-5718-1974-0371020-3

McKenna, S. J. (1984). A method of computing the complex probability function and other related functions over the whole complex plane. Astrophys. Space Sci., 107(1), 71-83. http://doi.org/10.1007/BF00649615

Pagnini, G., \& Mainardi, F. (2010). Evolution equations for the probabilistic generalization of the Voigt profile function. J. Comput. Appl. Math., 233(6), 1590-1595. http://dx.doi.org/10.1016/j.cam.2008.04.040

Poppe, G. P. M., \& Wijers, C. M. J. (1990a). More efficient computation of the complex error function. ACM Transact. Math. Software, 16(1), 38-46. http://doi.org/10.1145/77626.77629

Poppe, G. P. M., \& Wijers, C. M. J. (1990b). Algorithm 680: evaluation of the complex error function. ACM Transact. Math. Software, 16(1), 47. http://doi.org/10.1145/77626.77630

Quine, B. M., \& Abrarov, S. M. (2013). Application of the spectrally integrated Voigt function to line-by-line radiative transfer modelling. J. Quantit. Spectrosc. Radiat. Transfer, 127, 37-48. http://doi.org/10.1016/j.jqsrt.2013.04.020

Rybicki, G. B. (1989). Dawson's integral and the sampling theorem, Comp. Phys., 3, 85-87. http://dx.doi.org/10.1063/1.4822832

Schreier, F. (1992). The Voigt and complex error function: A comparison of computational methods. J. Quant. Spectrosc. Radiat. Transfer, 48(5, 6), 743-762. http://doi.org/10.1016/0022-4073(92)90139-U 
Sonnenschein, V., Raeder, S., Hakimi, A., Moore, I. D., \& Wendt, K. (2012). Determination of the ground-state hyperfine structure in neutral ${ }^{229}$ Th. J. Phys. B: At. Mol. Opt. Phys. 45(16), 165005. http://dx.doi.org/10.1088/0953-4075/45/16/165005

Srivastava, H. M., \& Chen, M. P. (1992). Some unified presentations of the Voigt functions. Astrophys. Space Sci., 192(1), 63-74. http://doi.org/10.1007/BF00653260

Weideman, J. A. C. (1994). Computation of the complex error function. SIAM J. Numer. Anal., 31(5), 1497-1518. http://dx.doi.org/10.1137/0731077

Zaghloul, M. R., \& Ali, A. N. (2011). Algorithm 916: computing the Faddeyeva and Voigt functions. ACM Transactions on Mathematical Software, 38(2), 15:1-15:22. http://dx.doi.org/10.1145/2049673.2049679

\section{Copyrights}

Copyright for this article is retained by the author(s), with first publication rights granted to the journal.

This is an open-access article distributed under the terms and conditions of the Creative Commons Attribution license (http://creativecommons.org/licenses/by/3.0/). 